\title{
Computation of Integrals With Oscillatory and Singular Integrands
}

\author{
By Bing-Yuan Ting and Yudell L. Luke
}

\begin{abstract}
This paper is concerned with evaluation of integrals whose integrands are oscillatory and contain singularities at the endpoints of the interval of integration. A typical form is $G(\theta)=\int_{a}^{b} w(x) e^{i \theta x} f(x) d x$, where $a$ and $b$ can be finite or infinite, $\theta$ is a parameter which is usually large, $f(x)$ is analytic in the range of integration, and the singularities are encompassed in the weight function $w(x)$. We suppose that $f(x)$ can be expanded in series of polynomials which are orthogonal over the interval of integration with respect to $w(x)$. There are two such expansions for $f(x)$. One is an infinite series which follows from the usual orthogonality property. The other is a polynomial approximation plus a remainder. The relations between the coefficients in these representations are detailed and methods for the evaluation of these are analyzed. Error analyses are provided. A numerical example is given to illustrate the effectiveness of the schemes developed.
\end{abstract}

1. Introduction. Evaluation of

$$
\int_{a}^{b} e^{i \theta x} f(x) d x
$$

with finite or infinite limits has wide applications in physical and engineering sciences. Here $\theta$ is usually large and $f(x)$ is sufficiently smooth, usually analytic, in the range of integration. When $\theta$ is large, use of ordinary integration rules which depend on tabulated values of $f(x) e^{i \theta x}$ is not efficient since many points need to be calculated owing to the highly oscillatory character of the integrand.

The earliest treatment of the integral is probably due to Filon [1] who approximates $f(x)$ by second-order polynomials over an even number of subintervals. Approximation of the integral follows by evaluating the moments $\int_{a_{1}}^{a_{1+2}} x^{k} e^{i \theta x} d x$, where $a_{i}=a+i h, h=(b-a) / 2 n, i=0,1, \ldots, 2 n-2$, and $k=0,1,2$. This method has been extended by Luke [2] who approximates the function $f(x)$ by a polynomial of at most tenth degree. The method can be viewed as an extended Newton-Cotes integration formula to which it reduces when $\boldsymbol{\theta}=0$. A considerable literature has been developed on the subject, and we make no attempt to survey it. But in this connection, see the papers by Lyness [3], [4], [5].

In this paper we study

$$
G(\theta)=\int_{a}^{b} w(x) e^{i \theta x} f(x) d x,
$$

where $a, b, \theta$, and $f(x)$ are as in (1.1) and where $w(x)$ is a weight function which reflects singularities at the endpoints of integration. For example, we might have

Received March 13, 1980; revised October 17, 1980.

1980 Mathematics Subject Classification. Primary 42A10, 65D30; Secondary 41A10.

Key words and phrases. Fourier coefficients, numerical quadrature, integration of oscillatory and singular integrands, interpolation. 
$a=-1, b=1, w(x)=(1-x)^{\alpha}(1+x)^{\beta}$, where $\alpha>-1, \beta>-1$. The idea is to expand $f(x)$ in series of polynomials $q_{n}(x)$ which are orthogonal over the interval of integration with respect to the weight function $w(x)$. Then forms for evaluating $G(\theta)$ readily follow by invoking orthogonality. There are two such expansions for $f(x)$. One is an infinite series which follows from the usual orthogonality property. The other is a polynomial approximation plus a remainder. Relations between the coefficients in these representations have been given by Luke [6]. See also Luke, Ting and Kemp [7]. These data are then employed to assess the error in the finite series approximation. Other properties and techniques pertinent to the computational process are developed. It calls for remark that we could also consider the situation where $e^{i \theta x}$ is expressed as a finite sum of the polynomials $q_{n}(x)$ plus a remainder. However, this is not done, for in most applications the infinite series expansion in the polynomials $q_{n}(x)$ is easily obtained.

In Section 2 we set down some basic equations for orthogonal polynomials needed in our work and give two formulas for the evaluation of (1.2). In Section 3 we apply the general scheme of Section 2 to the situation where the pertinent orthogonal polynomials are those of Jacobi. In Section 4 we give a numerical example to illustrate the effectiveness of the schemes developed. In Section 5 we discuss some other methods for the computation of (1.2) developed by Bakhvalov and Vasiléva [8], Piessens and Poleunis [9], Patterson [10], and Littlewood and Zakian [11]. In Section 6 we consider the situation where the range of integration is infinite and the orthogonal polynomials are those of Laguerre and Hermite.

2. Orthogonal Polynomials and the Evaluation of Oscillatory Integrals with Singularities. In this section we first set down some well-known results from the theory of orthogonal polynomials needed in our work. Let

$$
q_{n}(x)=\sum_{k=0}^{n} a_{k, n} x^{k}
$$

be a set of polynomials orthogonal over the interval $[a, b]$ with respect to the nonnegative weight function $w(x), w(x)>0$, so that

$$
\int_{a}^{b} w(x) q_{m}(x) q_{n}(x) d x=h_{n} \delta_{m n}
$$

where $\delta_{m n}$ is the usual notation for the Kronecker delta function. That is,

$$
\delta_{m n}=1 \quad \text { if } m=n, \quad \delta_{m n}=0 \text { if } m \neq n .
$$

The polynomials $q_{n}(x)$ satisfy the three-term recurrence relation

$$
\begin{aligned}
q_{n+1}(x) & =\left(A_{n} x+B_{n}\right) q_{n}(x)-C_{n} q_{n-1}(x), \quad n>0 \\
q_{1}(x) & =\left(A_{0} x+B_{0}\right) q_{0}(x),
\end{aligned}
$$

where

$$
\begin{array}{cc}
A_{n}=\frac{a_{n+1, n+1}}{a_{n, n}} ; & C_{n}=\frac{A_{n} h_{n}}{A_{n-1} h_{n-1}}, \quad n>0 ; \\
B_{n}=A_{n}\left(r_{n+1}-r_{n}\right) ; & r_{n}=\frac{a_{n-1, n}}{a_{n, n}}, \quad n>0, r_{0}=0 .
\end{array}
$$


We suppose that $f(x)$ can be expanded in a series of orthogonal polynomials $\left\{q_{n}(x)\right\}$ which is uniformly convergent in $[a, b]$. Thus

$$
\begin{aligned}
f(x) & =\sum_{k=0}^{\infty} c_{k} q_{k}(x), \\
c_{k} & =h_{k}^{-1} \int_{a}^{b} w(x) q_{k}(x) f(x) d x .
\end{aligned}
$$

Let $f_{n}(x)$ be the Lagrangian interpolating polynomial to $f(x)$ of degree $n$ with nodes at the zeros of $q_{n+1}(x)$, i.e., $f\left(x_{r}\right)=f_{n}\left(x_{r}\right), q_{n+1}\left(x_{r}\right)=0, r=0,1,2, \ldots, n$. It can be shown that

$$
\begin{aligned}
f_{n}(x) & =\sum_{k=0}^{n} d_{k, n} q_{k}(x), \\
d_{k, n} & =\frac{A_{n} h_{n}}{h_{k}} \sum_{r=0}^{n} \frac{f\left(x_{r}\right) q_{k}\left(x_{r}\right)}{q_{n+1}^{\prime}\left(x_{r}\right) q_{n}\left(x_{r}\right)},
\end{aligned}
$$

and the connection between the coefficients $c_{k}$ and $d_{k, n}$ is given by

$$
d_{k, n}=c_{k}+\sum_{s=0}^{\infty} c_{2 n+2+s-k} V_{2 n+2+s-k, k}^{(n)}
$$

where

$$
V_{j, k}^{(n)}=\frac{A_{n} h_{n}}{h_{k}} \sum_{r=0}^{n} \frac{q_{j}\left(x_{r}\right) q_{k}\left(x_{r}\right)}{q_{n+1}^{\prime}\left(x_{r}\right) q_{n}\left(x_{r}\right)}, \quad q_{n+1}\left(x_{r}\right)=0, r=0,1,2, \ldots, n,
$$

and

$$
V_{j, k}^{(n)}=\delta_{j k} \quad \text { for } j<n, k<n .
$$

We point out that the formula for $d_{k, n}$ is the Gaussian quadrature formula of the integral representation for $c_{k}$. In Gaussian quadrature, it is known that the coefficients of $f\left(x_{r}\right)$ must be positive. In particular, since $A_{n}$ can always be taken as positive, we must have $q_{n+1}^{\prime}\left(x_{r}\right) q_{n}\left(x_{r}\right)$ positive. The orthogonality relation (2.13) can be deduced from the Gaussian quadrature formula just noted. For details and further properties of $V_{j, k}^{(n)}$, including a recursion formula, see Luke [6].

After the manner of proof for the convergence of Gauss quadrature, see Davis and Rabinowitz [12], it can be shown that if $c_{k}$ and $d_{k, n}$ are defined as in (2.8) and (2.11), respectively, then, for $k$ fixed,

$$
\lim _{n \rightarrow \infty} d_{k, n}=c_{k}
$$

The error in approximating $f(x)$ by $f_{n}(x)$ is given by

$$
\begin{array}{r}
R_{n+1}(x)=f(x)-f_{n}(x)=c_{n+1} q_{n+1}(x)+c_{n+2}\left[q_{n+2}(x)+C_{n+1} q_{n}(x)\right] \\
+c_{n+3}\left[q_{n+3}(x)+\frac{A_{n+2} A_{n+1} h_{n+1}}{A_{n} A_{n-1} h_{n-1}} q_{n-1}(x)\right. \\
\left.\quad-C_{n+1}\left(\frac{A_{n+2} B_{n}}{A_{n}}-B_{n+2}\right) q_{n}(x)\right]
\end{array}
$$


or

$$
R_{n+1}(x)=c_{n+1} q_{n+1}(x)\left[1+\frac{c_{n+2}}{c_{n+1}}\left(A_{n+1} x+B_{n+1}\right)+\ldots\right] .
$$

In practice (2.14) and (2.15) can be advantageous, for even though the $c_{k}$ 's may be difficult to evaluate, asymptotic estimates are often available, see Donaldson and Elliott [13], and these can be used to appraise the remainder. In this instance only the first term or possibly first two terms of these equations can be used, at best, unless rather precise knowledge of the remainders in the asymptotic formulas for $c_{k}$ are known.

We now present two schemes for the evaluation of

$$
G(\theta)=\int_{a}^{b} w(x) e^{i \theta x} f(x) d x,
$$

where $a$ and $b$ are finite. We suppose that $f(x)$ is analytic in $[a, b]$. Assume first that $f(x)$ can be expanded in an infinite series of orthogonal polynomials $\left\{q_{n}(x)\right\}$ which is uniformly convergent in $[a, b]$. Here $w(x)$ is the weight function associated with the orthogonal polynomials $\left\{q_{n}(x)\right\}$. Thus $w(x)$ is positive and integrable in $[a, b]$ and encompasses the singularities of integrand in (2.16). The pertinent expansion formula for $f(x)$ has already been given by (2.7). Put the latter in (2.16). Then

$$
\begin{gathered}
G(\theta)=\sum_{k=0}^{\infty} c_{k} h_{k} b_{k}(\theta), \\
h_{k} b_{k}(\theta)=\int_{a}^{b} w(x) q_{k}(x) e^{i \theta x} d x .
\end{gathered}
$$

It should be noted that $b_{k}(\theta)$ is simply the coefficient of $q_{k}(x)$ in the expansion

$$
e^{i \theta x}=\sum_{k=0}^{\infty} b_{k}(\theta) q_{k}(x)
$$

For a finite series approximation to $G(\theta)$, consider

$$
G_{n}(\theta)=\int_{a}^{b} w(x) e^{i \theta x} f_{n}(x) d x
$$

where $f_{n}(x)$ is the approximation to $f(x)$ given by (2.9), (2.10). Put the latter in (2.20). Then

$$
G_{n}(\theta)=\sum_{k=0}^{n} d_{k, n} h_{k} b_{k}(\theta)
$$

In (2.17) and (2.21), the quantities $c_{k} h_{k}, d_{k, n} h_{k}$, and $b_{k}(\theta)$ depend on the orthogonal polynomials, but aside from this, $c_{k} h_{k}$ and $d_{k, n} h_{k}$ depend only on $f(x)$ while $b_{k}(\theta)$ depends only on $\theta$. This is advantageous, for the convergence of (2.17) can be satisfactory if only at least one of the quantities $c_{k} h_{k}$ or $b_{k}(\theta)$ decreases with sufficient rapidity as $k$ increases. Similar remarks hold for (2.21).

It is clear that $(2.21)$ is exact if $f(x)$ is a polynomial of degree $<n$. We now prove that $G_{n}(\theta)$ converges to $G(\theta)$ as $n \rightarrow \infty$.

THEOREM 1. If $f(x)$ is analytic on the finite interval $[a, b]$, then

$$
\lim _{n \rightarrow \infty} G_{n}(\theta)=G(\theta) \text {. }
$$


This is an easy consequence of a known result concerning the uniform convergence of the interpolating polynomials (based on zeros of orthogonal polynomials) in the case of functions $f(x)$ analytic on $[a, b]$; see, e.g., Freud [15, Theorem 8.1]. Using this theorem, we have

$$
\begin{aligned}
\left|G(\theta)-G_{n}(\theta)\right| & =\left|\int_{a}^{b} w(x) e^{i \theta x}\left(f(x)-f_{n}(x)\right) d x\right| \\
& \leqslant \int_{a}^{b} w(x)\left|f(x)-f_{n}(x)\right| d x<\mu_{0}\left\|f-f_{n}\right\|_{L_{\infty}[a, b]},
\end{aligned}
$$

where $\mu_{0}=\int_{a}^{b} w(x) d x$, and $\left\|f-f_{n}\right\|_{L_{\infty}[a, b]} \rightarrow 0$ by the above-mentioned theorem.

Next, we give a series representation for the error

$$
E_{n+1}(\theta)=G(\theta)-G_{n}(\theta) .
$$

Using (2.17), (2.21), and (2.14), we have

$$
\begin{aligned}
E_{n+1}(\theta)= & \int_{a}^{b} w(x) e^{i \theta x}\left[f(x)-f_{n}(x)\right] d x=\int_{a}^{b} w(x) e^{i \theta x} R_{n+1}(x) d x \\
= & c_{n+1} h_{n+1} b_{n+1}(\theta)+c_{n+2}\left[h_{n+2} b_{n+2}(\theta)+C_{n+1} h_{n} b_{n}(\theta)\right] \\
& +c_{n+3}\left[h_{n+3} b_{n+3}(\theta)+\frac{A_{n+2} A_{n+1} h_{n+1}}{A_{n} A_{n-1} h_{n-1}} b_{n-1}(\theta)\right. \\
& \left.\quad-C_{n+1}\left(\frac{A_{n+2} B_{n}}{A_{n}}-B_{n+2}\right) h_{n} b_{n}(\theta)\right] \\
& +\ldots
\end{aligned}
$$

Our discussion of (2.25) is much like that for (2.14) in that only one or possibly two terms of the formula will be useful in practice, unless realistic error bounds are available for the asymptotic representations of $c_{k}$. There is the further complication that convergence of (2.25) might be slow. In practice, it is suggested that one evaluates $G_{r}(\theta)$ for $r=n-2, n-1, n, \ldots$, and then uses an extrapolation technique, say Aitken's $\delta^{2}$-process [16].

3. Integration of Oscillatory Integrands With Algebraic Singularities. In the following we formulate the general scheme of Section 2 for the evaluation of the integral

$$
G(\theta)=\int_{-1}^{1} w(x) e^{i \theta x} f(x) d x
$$

where

$$
w(x)=(1-x)^{\alpha}(1+x)^{\beta}, \quad \alpha>-1, \beta>-1 .
$$

Here the pertinent orthogonal polynomials are those of Jacobi, notated as $P_{n}^{(\alpha, \beta)}(x)$.

In Section 2 we assumed that $f(x)$ is analytic in $[-1,1]$ and that it could be expanded in a series of the orthogonal polynomials $\left\{q_{n}(x)\right\}$ and that this series converges uniformly to $f(x)$ in $[-1,1]$. In the case of Jacobi polynomials, the analytic restriction can be relaxed in virtue of theorems by Natanson [17] and Prasad [18].

The pertinent definitions and results needed for Jacobi polynomials can be found in Erdélyi et al. [19] and Luke [6]. The formulas for $f(x), c_{k}, f_{n}(x), d_{k, n}$ etc. follow from the corresponding expressions in Section 2. When the orthogonal 
polynomials are those of Jacobi, some rather elegant representations for $b_{k}(\theta)$ can be given. From the work of Luke [20, Vol. 2, p. 29, Eq. (1)],

$$
b_{k}(\theta)=\frac{e^{-i \theta}(2 i \theta)^{k}}{(k+\lambda)_{k}} F_{1}(k+\beta+1 ; 2 k+\lambda+1 ; 2 i \theta), \quad \lambda=\alpha+\beta+1,
$$

and from this same reference, p. 48, Eq. (8),

$$
\begin{aligned}
b_{k}(\theta)= & \frac{i^{k} 2^{2 k+\alpha+1 / 2} \Gamma(k+\alpha+1 / 2)}{\theta^{\alpha+1 / 2}(k+\lambda)_{k}} \\
& \cdot \sum_{m=0}^{\infty} \frac{(-i)^{m}(m+k+\alpha+1 / 2)(2 k+2 \alpha+1)_{m}(\alpha-\beta)_{m}}{(2 k+\lambda+1)_{m} m !} \\
& \cdot J_{m+k+\alpha+1 / 2}(\theta) .
\end{aligned}
$$

The latter can be simplified considerably if $\alpha=\beta$ whence $\lambda=2 \alpha+1$. In this instance

$$
b_{k}(\theta)=\frac{i^{k} \pi^{1 / 2}(2 k+2 \alpha+1) \Gamma(k+2 \alpha+1)}{(2 \theta)^{\alpha+1 / 2} \Gamma(k+\alpha+1)} J_{k+\alpha+1 / 2}(\theta) .
$$

If $\alpha=0,-\frac{1}{2}$ and $\frac{1}{2}$ in (3.5), we get the cases of Legendre, Chebyshev of the first kind and of the second kind, respectively. Thus

$$
\begin{aligned}
& b_{k}(\theta)=\frac{i^{k} \pi^{1 / 2}(2 k+1)}{(2 \theta)^{1 / 2}} J_{k+1 / 2}(\theta) \quad \text { (Legendre), } \\
& b_{k}(\theta)=\frac{2 \varepsilon_{k} i^{k} k !}{(1 / 2)_{k}} J_{k}(\theta), \quad \varepsilon_{0}=\frac{1}{2}, \varepsilon_{k}=1, k>0
\end{aligned}
$$

$$
b_{k}(\theta)=\frac{2 i^{k}(k+1)(k+1) !}{\theta(3 / 2)_{k}} J_{k+1}(\theta)
$$

(Chebyshev of the second kind).

Equations (3.6)-(3.8) are the same as those given by Patterson [10].

The recursion formula for $b_{k}(\theta)$,

$$
\begin{aligned}
b_{k+1}(\theta)= & \frac{(k+\lambda)(2 k+\lambda+2)}{(k+\alpha+1)(k+\beta+1)(2 k+\lambda-1)} \\
& \cdot\left[(\alpha-\beta)+\frac{i(2 k+\lambda-1)(2 k+\lambda+1)}{2 \theta}\right] b_{k}(\theta) \\
& +\frac{(k+\lambda-1)(k+\lambda)(2 k+\lambda+1)(2 k+\lambda+2)}{(k+\alpha+1)(k+\beta+1)(2 k+\lambda-2)(2 k+\lambda-1)} b_{k-1}(\theta),
\end{aligned}
$$

follows from Luke [20, Vol. 2, p. 157, Eqs. (17), (18) and p. 153, Eq. (4)].

If $\alpha=\beta, \lambda=2 \alpha+1$, then (3.9) simplifies to

$$
\begin{aligned}
b_{k+1}(\theta)= & \frac{i(k+2 \alpha+1)(2 k+2 \alpha+3)}{(k+\alpha+1) \theta} b_{k}(\theta) \\
& +\frac{(k+2 \alpha)(k+2 \alpha+1)(2 k+2 \alpha+3)}{(k+\alpha)(k+\alpha+1)(2 k+2 \alpha-1)} b_{k-1}(\theta),
\end{aligned}
$$

which is obviously akin to the recursion formula for $J_{\nu}(x)$ in view of (3.6)-(3.8). 
With $x=1$ and $x=-1$ in the expansion for $e^{i \theta x}$, see (2.19), we get the respective normalization relations

$$
\begin{aligned}
\sum_{k=0}^{\infty} \frac{(\alpha+1)_{k}}{k !} b_{k}(\theta) & =e^{i \theta} \\
\sum_{k=0}^{\infty} \frac{(-)^{k}(\beta+1)_{k}}{k !} b_{k}(\theta) & =e^{-i \theta}
\end{aligned}
$$

If $\alpha=\beta, \lambda=2 \alpha+1$, then put $x=0$ in the expansion for $e^{i \theta x}$ and obtain

$$
\sum_{k=0}^{\infty} \frac{(-)^{k} \Gamma\left(k+\frac{1}{2}\right) \Gamma(2 k+\alpha+1)}{\pi^{1 / 2} \Gamma(k+\alpha+1)(2 k) !} b_{2 k}(\theta)=1
$$

We now turn to the evaluation of the coefficients $b_{k}(\theta)$ by use of the recurrence formula used in the backward direction together with one of the normalization relations (3.11)-(3.13), as appropriate.

This technique, also known as the Miller algorithm, proceeds as follows. Consider the second-order difference equation

$$
y(n)+C_{1}(n) y(n+1)+C_{2}(n) y(n+2)=0, \quad C_{1}(n), C_{2}(n) \neq 0
$$

where $n$ is an integer $>0$. Let $N$ be a large positive integer. Put

$$
\Lambda_{N+1}(N)=0, \quad \Lambda_{N}(N)=1,
$$

and calculate $\Lambda_{n}(N)$ for $0<n<N-1$ from (3.14) with $y(n)$ replaced by $\Lambda_{n}(N)$, i.e.,

$$
\Lambda_{n}(N)+C_{1}(n) \Lambda_{n+1}(N)+C_{2}(n) \Lambda_{n+2}(N)=0 .
$$

Suppose we are given the convergent series (called a normalization relationship)

$$
1=\sum_{k=0}^{\infty} L(k) y_{1}(k)
$$

where $y_{1}(k)$ is the desired solution of (3.14). Define

$$
\Omega(N)=\sum_{k=0}^{N} L(k) \Lambda_{k}(N)
$$

and

$$
\Gamma_{n}(N)=\Lambda_{n}(N) / \Omega(N) .
$$

Notice that it is no loss of generality to assume the sum (3.16) is 1 , since $L(k)$ can always be so normalized. The Miller algorithm is then described by the following theorem due to Gautschi [21].

THEOREM 2. Let there exist a solution of (3.14), $y_{2}(n)$, which is linearly independent of $y_{1}(n)$ with the property that $y_{2}(n)$ is not zero for $n$ sufficiently large, and

$$
\lim _{n \rightarrow \infty} \frac{y_{1}(n)}{y_{2}(n)}=0, \quad \lim _{n \rightarrow \infty} \frac{y_{1}(n+1)}{y_{2}(n+1)} \sum_{k=0}^{n} L(k) y_{2}(k)=0 .
$$

Then

$$
\lim _{N \rightarrow \infty} \Gamma_{n}(N)=y_{1}(n), \quad n=0,1,2, \ldots
$$

When the latter holds, we say that the backward recursion process is convergent. 
If $y_{1}(0)$ can be readily computed, and $y_{1}(0) \neq 0$, then we can use this value as a normalization relation. In this event, (3.16) holds with $L(k)=0$ for $k>1$, and $L(0)=1 / y_{1}(0)$. Then in place of Theorem 2 , we have the

COROLlaRY. Let $y_{2}(n)$ be as in Theorem 2 but with (3.19) amended to read

$$
\lim _{n \rightarrow \infty} \frac{y_{1}(n)}{y_{2}(n)}=0 \text {. }
$$

Then

$$
\lim _{N \rightarrow \infty} \frac{\Lambda_{n}(N)}{\Lambda_{0}(N)} y_{1}(0)=y_{1}(n), \quad n=0,1,2, \ldots
$$

Proof that the backward recurrence process converges for the case at hand is readily established by examining the Puiseux diagram of (3.9), see Gautschi [21], which shows that (3.9) has a solution that grows rapidly to $\infty$ and another one that grows equally rapidly to 0 as $k \rightarrow \infty$. It suffices to show, therefore, that $b_{k}(\theta) \rightarrow 0$ as $k \rightarrow \infty$ (or only that $b_{k}(\theta)$ is bounded). If $f(x)$ is analytic in a domain containing $[-1,1]$, this follows from Cauchy's formula

$$
\int_{-1}^{1} w(x) q_{k}(x) f(x) d x=\frac{1}{2 \pi i} \oint f(z) \int_{-1}^{1} \frac{w(x) q_{k}(x) d x}{z-x},
$$

for, as is well known, the inner integral tends to zero geometrically as $k \rightarrow \infty$. Since $h_{k}^{-1}=O(k)$ as $k \rightarrow \infty$, it follows that $b_{k}(\theta)$, see (2.18), tends to zero as $k \rightarrow \infty$. Proof of the results corresponding to the second condition in (3.19) for any of the normalizations (3.11), (3.12) is straightforward and we omit the details.

An alternative proof concerning the behavior of the two solutions of (3.9) can also be deduced by solution of the difference equation after the manner discussed by Wimp [22], [23]. Further, an alternative technique for the evaluation of $b_{k}(\theta)$ according to (3.3) can be deduced from a result given by Luke [24]. This analysis leads to the statement

$$
b_{k}(\theta)=2^{\frac{1}{2}-\lambda}(i e \theta / k)^{k}\left[1+O\left(k^{-1}\right)\right], \quad k \rightarrow \infty .
$$

Lyness [5] studied the evaluation of (3.1). No use is made of orthogonality. His approach is an extension of the technique developed in Lyness [3], [4] to evaluate (3.1) when $w(x)=1$. In the latter papers, trapezoidal sums of the form $\sum_{i=0}^{r} f\left(x_{i}\right)$ and values of $f^{\prime}\left(x_{i}\right)$ at $x=-1$ and $x=1$ are required. These same features carry over to the case $w(x) \neq 1$. In the more general case, values of the generalized zeta function $\zeta(s, y)$ are also needed. The results are quite complicated and not as simple as the approach of this paper.

4. Numerics. In this section we present a numerical example to illustrate the effectiveness of the schemes developed in this paper. Consider the integral

$$
G(\theta)=\int_{0}^{1} \frac{e^{i \theta x}}{x^{1 / 2}(1+x)} d x .
$$

Notice that the integrand contains a square root singularity at $x=0$. In the notations of the previous section $\alpha=0$ and $\beta=-\frac{1}{2}$. From the work of Luke [20, Vol. 2, p. 31, Eq. (1)], 


$$
\begin{gathered}
(1+x)^{-1}=\sum_{k=0}^{\infty} c_{k} R_{k}^{(\alpha, \beta)}(x), \\
c_{k}=\frac{(-)^{k} k !}{(k+\lambda)_{k}}{ }_{2} F_{1}(k+\beta+1, k+1 ; 2 k+\lambda+1 ;-1), \quad \lambda=\alpha+\beta+1,
\end{gathered}
$$

where $R_{k}^{(\alpha, \beta)}(x)=P_{k}^{(\alpha, \beta)}(2 x-1)$ is the shifted Jacobi polynomial. Also from the same reference p. 159, Eqs. (26)-(28),

$$
\begin{gathered}
(k+1) c_{k}+\frac{2 k+\lambda}{(k+\lambda)(2 k+\lambda+3)}\{(k+2)(k+\beta+2)(2 k+\lambda+1) \\
-(k+1)(k+\beta+1)(2 k+\lambda+3) \\
+(2 k+\lambda+1)(2 k+\lambda+3)\} c_{k+1} \\
+\frac{(2 k+\lambda)(2 k+\lambda+1)(k+\alpha+2)(k+\beta+2)}{(k+\lambda)(2 k+\lambda+3)(2 k+\lambda+4)} c_{k+2}=0 .
\end{gathered}
$$

Put $x=0$ in (4.2) to get the normalization relation

$$
\sum_{k=0}^{\infty} \frac{(-)^{k}(\beta+1)_{k}}{k !} c_{k}=1,
$$

which can be effectively used with (4.3) in the backward direction to generate the $c_{k}$ 's. These data for $\alpha=0$ and $\beta=-\frac{1}{2}$ and $N=15$ are presented in column $A$ of Table 1. Again, from the work of Donaldson and Elliott [13], we have

$$
c_{k} \sim(-)^{k}(\pi k)^{1 / 2} 2^{3 / 4+\alpha / 2} u^{2 k+\lambda}, \quad u=2^{1 / 2}-1 .
$$

Numerical values of $c_{k}$ based on this formula with $\alpha=0$ and $\beta=-\frac{1}{2}$ are posted in column B of Table 1 . In Table 2 the values of $b_{k}(\theta)$, as developed by use of the backward recursion scheme, are posted.

TABLE 1

The coefficients $c_{k}$

\begin{tabular}{lrcc}
\hline & \multicolumn{1}{c}{$A$} & $c_{k}$ & $B$ \\
\hline 0 & 0.7853981634 & - \\
1 & -0.3539816340 & -0.3291608838 \\
2 & 0.0829594999 & 0.0798678230 \\
3 & -0.0172210508 & -0.0167828652 \\
4 & 0.0033904936 & 0.0033249422 \\
5 & -0.0006479078 & -0.0006378047 \\
6 & 0.0001214617 & 0.0001198746 \\
7 & -0.0000224678 & -0.0000222151 \\
8 & 0.0000041153 & 0.0000040747 \\
9 & -0.0000007481 & -0.0000007415 \\
10 & 0.0000001352 & 0.0000001341 \\
11 & -0.0000000243 & -0.0000000241 \\
12 & 0.0000000043 & 0.0000000043 \\
13 & -0.0000000008 & -0.0000000008 \\
14 & 0.0000000001 & 0.0000000001 \\
\hline
\end{tabular}


TABLE 2

The coefficients $b_{k}(\theta)$ by use of the backward recursion method $\alpha=0, \beta=-\frac{1}{2}, \theta=4$ and $N=15$

\begin{tabular}{|c|c|c|}
\hline \multirow[b]{2}{*}{$k$} & \multicolumn{2}{|c|}{$b_{k}(\theta)$} \\
\hline & Real & Imaginary \\
\hline 0 & 0.2307307312 & 0.4023882447 \\
\hline 1 & $-1.66355 \quad 31 \div 58$ & -0.1768696565 \\
\hline 2 & 0.3118723552 & -1.2952637469 \\
\hline 3 & 0.5569064306 & 0.1668853441 \\
\hline 4 & -0.0552823417 & 0.1659848931 \\
\hline 5 & -0.0379278179 & -0.0134516322 \\
\hline 6 & 0.0026012917 & -0.0070338286 \\
\hline 7 & 0.0010982646 & 0.0004185123 \\
\hline 8 & -0.0000577316 & 0.0001481282 \\
\hline 9 & -0.0000175877 & -0.0000069760 \\
\hline 10 & 0.0000007503 & -0.0000018653 \\
\hline ג1 & 0.0000001788 & 0.0000000727 \\
\hline 12 & -0.0000000064 & 0.0000000156 \\
\hline 13 & -0.0000000013 & -0.0000000005 \\
\hline 14 & 0.0000000000 & -0.0000000001 \\
\hline
\end{tabular}

As previously noted, $b_{k}(\theta)$ can be easily calculated from a series given by Luke [24]. With $\theta=4,20$ terms of this series gave essentially the same values in Table 2. Substituting the values of $c_{k}$ and $b_{k}(\theta)$ in the formula (2.17) and summing the first twelve terms with $\alpha=0, \beta=-\frac{1}{2}$ and $\theta=4$, we get

$$
G(\theta)=0.6022343648+0.6328594815 i \text {. }
$$

Next we calculate the zeros of $R_{n+1}^{(0,-1 / 2)}(x)$ and the $d_{k, n}$ 's which are needed to compute the approximation $G_{n}(\theta)$ in $(2.21)$ as well as the error estimates $E_{n+1}(\theta)$ in (2.25) for $n=3$. We post these data in the following tables.

\section{TABLE 3}

Zeros of $R_{n+1}^{(0,-1 / 2)}(x)$ and values of $d_{k, n}$, and $G_{n}(\theta), n=3, \theta=4$

\begin{tabular}{llr}
\hline$k$ & \multicolumn{1}{c}{$x_{k}$} & \multicolumn{1}{c}{$o_{k, n}$} \\
\hline 0 & 0.0336482681 & 0.7853972062 \\
1 & 0.2761843139 & -0.3539720627 \\
2 & 0.6346774762 & 0.0828862799 \\
3 & 0.9221566085 & -0.0167109435 \\
$G_{3}(\theta)=0.602285875+0.63282523791$ & \\
$E_{\text {rror }}=-0.515\left(10^{-4}\right)+0.342\left(10^{-4}\right) 1$. & \\
\hline
\end{tabular}


It is of interest to know the convergence properties of the error representation (2.25) for our example. This is readily done since the $c_{k}$ 's are known rather precisely. In the following table we illustrate computation of $E_{n+1}(\theta)$ using $1,2,3$ and 4 terms.

\section{TABLE 4}

The error estimates $E_{n+1}(\theta), n=3, \theta=4$

\begin{tabular}{cc}
\hline No. of Terms & $E_{n+1}(\theta)$ \\
\hline 1 & $-0.221\left(10^{-4}\right)+0.622\left(10^{-4}\right) 1$ \\
2 & $-0.634\left(10^{-4}\right)+0.539\left(10^{-4}\right) 1$ \\
3 & $-0.583\left(10^{-4}\right)+0.327\left(10^{-4}\right) 1$ \\
4 & $-0.510\left(10^{-4}\right)+0.335\left(10^{-4}\right) i$
\end{tabular}

It is clear that at best three terms give a fair appraisal of the accuracy. As previously noted, in practice the $c_{k}$ 's are usually not known. Even if known asymptotically, only one or possibly two terms of (2.25) can be used unless rather precise information is available on the remainders in the asymptotic formulas. As indicated by the example, use of only one term gives at best an order of magnitude estimate of the error. Further, convergence of (2.25) is slow and use of (2.25) does not improve as $n$ increases. This indicates that one should have available one or more a posteriori approaches to estimate the error and improve convergence. That is, compute $G_{n}(\theta)$ for several successive values of $n$ and use these data to get estimates of the error and improve the accuracy of the approximations obtained, say by use of Aitken's $\delta^{2}$-process. This we presently do after tabulating further approximations for $G_{n}(\theta)$.

TABLE 5

$G_{n}(\theta)$ for various $n$ and the true errors

\begin{tabular}{|c|c|c|}
\hline & \multicolumn{2}{|c|}{$G_{n}(\theta)$} \\
\hline$\underline{n}$ & Beal & Imagtnary \\
\hline 3 & 0.6022858752 & 0.6328252379 \\
\hline 4 & 0.6022335930 & 0.6328577309 \\
\hline 5 & 0.6022343191 & 0.6328594967 \\
\hline 6 & 0.6022343651 & 0.6328594825 \\
\hline \multirow[t]{2}{*}{7} & 0.6022343648 & 0.6328594815 \\
\hline & \multicolumn{2}{|c|}{ True Error } \\
\hline$\underline{n}$ & Real & Imaginary \\
\hline 3 & $-0.515\left(10^{-1}\right)$ & $0.342\left(10^{-4}\right)$ \\
\hline 4 & $0.077\left(10^{-5}\right)$ & $0.175\left(10^{-5}\right)$ \\
\hline 5 & $0.455\left(10^{-7}\right)$ & $-0.153\left(10^{-7}\right)$ \\
\hline 6 & $-0.031\left(10^{-8}\right)$ & $-0.104\left(10^{-8}\right)$ \\
\hline 7 & 0 & 0 \\
\hline
\end{tabular}


Since $G_{n}(\theta)$ converges, an often useful indicator of the error in $G_{n}(\theta)$ is $G_{n+1}(\theta)-G_{n}(\theta)$. Let $N$ be the largest value of $n$ for which $G_{n}(\theta)$ is calculated. Then a better estimate of the error in $G_{n}(\theta)$ is $G_{N}(\theta)-G_{n}(\theta)$. Neither of these techniques can be guaranteed. However, they work pretty well in practice, especially if the sequence $G_{n}(\theta)$ converges with sufficient rapidity. This is the situation for the case at hand.

With the values of $G_{n}(\theta), n=3(1) 7$, we apply Aitken's $\delta^{2}$-process to three consecutive sums, $G_{n-2}(\theta), G_{n-1}(\theta)$ and $G_{n}(\theta)$ for $n=5(1) 7$. We post the results in Table 6.

TABLE 6

$G_{n}(\theta)$, improved values by application of the Aitken's

$\delta^{2}$-process to $\left\{G_{n}(\theta)\right\}$

\begin{tabular}{|c|c|c|}
\hline \multirow[b]{2}{*}{$\underline{n}$} & \multicolumn{2}{|c|}{$\bar{G}_{n}(\theta)$} \\
\hline & Real & Imaginary \\
\hline 5 & 0.6022343767 & 0.6328594817 \\
\hline 6 & 0.6022343648 & 0.6328594813 \\
\hline 7 & 0.6022343648 & 0.6328594815 \\
\hline
\end{tabular}

Further improvement is obtained by applying the $\delta^{2}$-process to the sequence $\left\{\bar{G}_{n}(\theta)\right\}$, etc. We omit the numerics.

5. Some Other Expansions. In this section we discuss some related but special expansions discussed by other workers.

Suppose we want to evaluate $\int_{-1}^{1} f(x) d x$, where $f(x)$ is continuous in $[-1,1]$, by the techniques previously considered. Here $\theta=0$ and the relevant orthogonal polynomials are those of Legendre since $w(x)=1$. In this event, we need the zeros $x_{\alpha}$ of $P_{n+1}(x), P_{n+1}^{\prime}\left(x_{\alpha}\right)$ etc. to compute the coefficients $d_{k, n}$. Actually only $d_{0, n}$ is required. To avoid evaluation of $x_{\alpha}$ and those quantities dependent on $x_{\alpha}$, the idea of Clenshaw and Curtis [14] is to approximate $f(x)$ by $f_{n}(x)$ where $f_{n}(x)$ is an interpolatory polynomial whose points of collocation are the zeros of the Chebyshev polynomial of the first kind $T_{n+1}(x)$, for in this situation the zeros and other needed data are simply expressed.

Some ideas of a similar character has been suggested for the evaluation of

$$
G(\theta)=\int_{-1}^{1} e^{i \theta x} f(x) d x .
$$

This is a special case of $(3.1)$ where $w(x)=1$. As previously noted, Bakhvalov and Vasiléva [8] express $f(x)$ in series of Legendre polynomials. In this event, evaluation of (5.1) obtains once we compute $b_{k}(\theta)$ by use of (3.6). In general, all of this has been generalized in Section 3.

Piessens and Poleunis [9] and Patterson [10] choose to express $f(x)$ in a series of Chebyshev polynomials of the first kind for the reasons previously stated. But in this event, one must know

$$
v_{k}(\theta)=\int_{-1}^{1} T_{k}(x) e^{i \theta x} d x
$$


to compute (5.1). Piessens and Polenius show that (5.2) can be expressed as an infinite series of Bessel functions. The coefficients $v_{k}(\theta)$ can be shown to satisfy a three-term inhomogeneous recurrence formula and this can be used to evaluate these numbers. Indeed, this notion can be generalized to get an inhomogeneous recurrence formula for the evaluation of

$$
W_{k}^{(\alpha, \beta)}(\theta)=\int_{-1}^{1} e^{i \theta x} P_{k}^{(\alpha, \beta)}(x) d x
$$

so that evaluation of (5.1) follows once the coefficients for the expansion of $f(x)$ in terms of $P_{k}^{(\alpha, \beta)}(x)$ are known. This recurrence formula is omitted here. It is given in a thesis by Ting [25].

Littlewood and Zakian [11] extend the methods of Piessens and Poleunis [9], and Patterson [10]. The idea is to approximate $f(x)$ by a finite series of Chebyshev polynomials of the first kind. Here the Chebyshev coefficients $d_{k, n}$ are easily determined. Now suppose we approximate $f(x)$ by a finite series of Legendre polynomials of the same degree as the Chebyshev case and equate these two series. Then we can express the Legendre coefficients $e_{k, n}$ in terms of $d_{k, n}$. Then evaluation of (5.1) is quite simple in view of (3.6). The main feature of this approach is to retain the advantage of the Chebyshev approximation for $f(x)$ and avoid the evaluation of infinite series of Bessel functions as noted above. The above approaches have merit only to the extent that $f(x)$ is approximated by a series of Chebyshev polynomials. However, there is little merit insofar as computations relating to $b_{k}(\theta)$ are concerned since in all cases one must compute Bessel functions. On this point, the authors preferred to suggest calculation of the same by use of the backward recursion scheme. But, we have shown that $b_{k}(\theta)$ can always be calculated in this manner for any $\alpha$ and $\beta$ greater than -1 .

In the above treatments $w(x)=1$. For arbitrary $w(x)$ of the form (3.2), the Littlewood and Zakian technique can be generalized to get another prescription to evaluate (2.20). The point is that given the coefficients in $\sum_{k=0}^{n} d_{k, n} T_{k}(x)$, we can derive a recursive process to get the coefficients in $\sum_{k=0}^{n} e_{k, n} P_{k}^{(\alpha, \beta)}(x)$. Then once we determine the appropriate $b_{k}(\theta)$, evaluation of $G_{n}(\theta)$ follows from (2.21). The schema to get the $e_{k, n}$ 's just noted will be the subject of another paper to be given elsewhere.

6. Oscillatory Integrals Over Infinite Range. In the following we consider evaluation of (1.2) where $a=0, b=\infty, w(x)=e^{-x} x$, and the analogous orthogonal polynomials are those of Laguerre (generalized), and where $a=-\infty, b=\infty$, $w(x)=e^{-x^{2}}$, and the corresponding polynomials are those of Hermite. All of our results are formal since convergence criteria are rather imprecise when compared with the corresponding Jacobi situation.

The generalized Laguerre polynomial can be defined as a limiting form of the Jacobi polynomial. Thus, all needed formulas can be deduced from the results for the latter polynomials by use of this confluence limit.

We consider

$$
G(\theta)=\int_{0}^{\infty} w(x) e^{i \theta x} f(x) d x
$$


where $f(x)$ is real, analytic in $(0, \infty)$ and for all sufficiently large values of $x$

$$
|f(x)|<\frac{e^{x}}{x^{\alpha+\rho+1}}, \text { for some } \rho>0 .
$$

The formulas for $c_{k}, d_{k, n}$ etc. follow from (2.8)-(2.11), while those for $G(\theta)$ and $G_{n}(\theta)$ come from (2.17) and (2.21). For the present case,

$$
\begin{aligned}
b_{k}(\theta) & =h_{k}^{-1} \int_{0}^{\infty} x^{\alpha} e^{-(1-i \theta) x} L_{k}^{(\alpha)}(x) d x \\
& =i^{\alpha+1} \theta^{k}\left(\frac{\theta-i}{1+\theta^{2}}\right)^{k+\alpha+1} .
\end{aligned}
$$

Quite often, the technique described in this section is poor because we are dealing with an infinite span and $f(x)$ may not have sufficient decay characteristics. For an example, see Patterson [10]. To improve the situation, Patterson suggests that we break the integral into the ranges $[0, a]$ and $[a, \infty]$, thus

$$
G(\theta)=\int_{0}^{a} x^{\alpha} e^{-x} e^{i \theta x} f(x) d x+\int_{a}^{\infty} x^{\alpha} e^{-x} e^{i \theta x} f(x) d x,
$$

or

$$
\begin{aligned}
G(\theta)= & (a / 2)^{\alpha+1} \exp \{-a(1-i \theta) / 2\} \int_{-1}^{1}(1+x)^{\alpha} e^{i a \theta / 2} f\left(\frac{a(1+x)}{2}\right) d x \\
& +\int_{0}^{\infty}(x+a)^{\alpha} e^{-(x+a)} e^{i \theta(x+a)} f(x+a) d x .
\end{aligned}
$$

The first integral can be evaluated by the scheme in Section 3 while the second integral is of the type considered in this section. Note that the decay properties of $f(x+a)$ are better than those of $f(x)$, but not materially so if $f(x)$ only decays algebraically. In practice, it is perhaps best to evaluate the infinite integral in (6.4) for large $a$ by asymptotic methods.

Hermite polynomials are but a special case of Laguerre polynomials. We record only the formula

$$
\begin{aligned}
b_{k}(\theta) & =h_{k}^{-1} \int_{-\infty}^{\infty} e^{-x^{2}} e^{i \theta x} H_{k}(x) d x \\
& =(i \theta / 2)^{k} \exp \left(-\theta^{2} / 4\right) / k ! .
\end{aligned}
$$

Acknowledgement. The authors are grateful to the referee for suggestions which improved the paper.

The Marley Cooling Tower Company

Mission, Kansas 66201

Department of Mathematics

University of Missouri at Kansas City

Kansas City, Missouri 64110

1. L. N. G. Filon, "On a quadrature formula for trigonometric integrals," Proc. Roy. Soc. Edinburgh, v. 49, 1929, pp. 38-47.

2. Y. L. Luxe, "On the computation of oscillatory integrals," Part 2, Proc. Cambridge Philos. Soc., v. 50, 1954, pp. 269-277.

3. J. N. LYNess, "The calculation of Fourier coefficients by Möbius inversion of the Poisson summation formula. Part I, Functions whose early derivatives are continuous," Math. Comp., v. 24, 1970, pp. 101-135. 
4. J. N. LYNEss, "The calculation of Fourier coefficients by the Möbius inversion of the Poisson summation formula. Part II, Piecewise continuous functions and functions with poles near [0, 1]," Math. Comp., v. 25, 1971, pp. 59-78.

5. J. N. LYNess, "The calculation of Fourier coefficients by the Möbius inversion of the Poisson summation formula. Part III, Functions having algebraic singularities," Math. Comp., v. 25, 1971, pp. 483-493.

6. Y. L. LUKe, "On the error in a certain interpolation formula and in the Gaussian integration formula," J. Austral. Math. Soc. Ser. A (2), v. 19, 1975, pp. 196-209.

7. Y. L. LUKe, B. Y. TING \& M. J. KeMP, “On generalized Gaussian quadrature," Math. Comp., v. 29, 1975, pp. 1083-1093.

8. N. S. Bakhvalov \& L. G. Vasiléva, "The calculation of integrals of oscillatory functions by interpolation of the Gaussian quadrature nodes," Z. Vycisl. Mat. i Mat. Fiz., v. 8, 1968, pp. 175-181.

9. R. Piessens \& F. Poleunis, "A numerical method for the integration of oscillatory functions," $B I T$, v. 11, 1971, pp. 317-327.

10. T. N. L. PAtrerson, "On higher precision methods for the evaluation of Fourier integrals with finite and infinite limits," Numer. Math., v. 27, 1976, pp. 41-52.

11. R. K. LITtLEWOod \& V. ZAKIAN, "Numerical evaluation of Fourier integrals," J. Inst. Math. Appl., v. 18, 1976, pp. 331-339.

12. P. J. DAvis \& P. RABinowitz, Numerical Integration, Blaisdell, Boston, Mass., 1967.

13. J. D. Donaldson \& D. Elliotr, “A uniform approach to quadrature rules with asymptotic estimates of their remainders," SIAM J. Numer. Anal., v. 9, 1972, pp. 573-602.

14. C. W. Clenshaw \& A. R. CuRTis, "A method for numerical integration on an automatic computer," Numer. Math., v. 2, 1960, pp. 197-205.

15. G. Freud, Orthogonal Polynomials, Pergamon Press, Oxford and New York, 1971.

16. A. C. Artken, "On Bernoulli's numerical solution of algebraic equations," Proc. Roy. Soc. Edinburgh, v. 46, 1926, pp. 289-305.

17. I. P. Natanson, Constructive Function Theory, Vols. 1, 2 and 3, Ungar, New York, 1964.

18. J. PraSAD, "On an approximation of a function and its derivatives," Publ. Inst. Math., v. 14 (28), 1972, pp. 129-132.

19. A. ERdély et AL., Higher Transcendental Functions, Vol. 2, McGraw-Hill, New York, 1954.

20. Y. L. LuKe, The Special Functions and Their Approximations, Vols. 1 and 2, Academic Press, New York and London, 1969.

21. W. GAUTSCHI, “Computational aspects of three-term recurrence relations," SIAM Rev., v. 9, 1967, pp. 24-82.

22. J. WnMP, On recursive computation, Report ARL 69-0186, Aerospace Res. Labs., Wright-Patterson Air Force Base, Ohio, 1969.

23. J. WIMP, "Recent development in recursive computation," SIAM Studies in Appl. Math., Vol. VI, Philadelphia, Pa., 1970, pp. 101-123.

24. Y. L. LUKE, "On the error in the Padé approximants for a form of the incomplete gamma function including the exponential function," SIAM J. Math. Anal., v. 6, 1975, pp. 829-839.

25. B. Y. TING, Evaluation of Integrals Whose Integrands Are Oscillatory and Singular, Ph.D. Thesis, University of Missouri, Kansas City, Mo., 1979. 